\title{
Long-term photometric behaviour of the RS CVn binary RT Lacertae
}

\author{
Ö. Çakırlı ${ }^{1}$, C. İbanoğlu ${ }^{1}$, G. Djurašević ${ }^{2}$, S. Erkapić ${ }^{2}$, S. Evren ${ }^{1}$, and G. Taş ${ }^{1}$
}

\author{
${ }^{1}$ Ege University Observatory, Bornova, İzmir, Turkey \\ 2 Astronomical Observatory, Volgina 7, 11160 Belgrade, Yugoslavia and Isaac Newton Institute of Chile, Yugoslav branch \\ Received 11 December 2002 / Accepted 24 April 2003
}

\begin{abstract}
A sequence of the seasonal light curves of RT Lac, covering the period 1978-2000, is analysed in the framework of the starspot hypothesis to define the spot distribution, based on the interpretation of the $B$-band observations. The analysis of the corresponding light curves is made using Djurašević's inverse-problem method. To explain the light-curve variations we modelled the binary system using a Roche model that involved regions containing spots on both components. Satisfactory fits were obtained assuming spots on both components. The more-massive G5 primary appears to be the most active star in the system and its spotted areas are mainly responsible for the light-curve distortions. Spots are concentrated around longitudes $45^{\circ}-170^{\circ}$ and at high latitudes (above $45^{\circ}$ ). Our analysis indicates two spots with diameters of $\sim 10^{\circ}-50^{\circ}$ on both hemispheres of the primary. However, the less-massive cool component seems to have only one spot which covers a relatively small area. Total spotted area of the more-massive primary component indicates clear evidence for a short-term activity cycle with a period of $8.4 \mathrm{yr}$, and a possible long-term cycle with a period of $33.5 \mathrm{yr}$. The G9IV secondary does not show any evidence for an activity cycle, its spot coverage appearing rather constant at about $10 \%$ of its surface. The variation of the orbital period seems to be correlated with the total activity level of the system. In particular, the decrement of the orbital period appears to be associated with minimum spottedness and sizeable changes of the surface spot pattern distribution on the surface of each star. This result, if confirmed by the future observations, can provide further support for recently proposed models for connection between the magnetic activity and orbital period variations.
\end{abstract}

Key words. stars: activity - binaries: close - stars: individual: RT Lacertae - starspots

\section{Introduction}

The relatively long-period eclipsing RS CVn-type binary RT Lac (=SAO 51563, G5IV+G9IV) has been the subject of numerous photometric studies in the last two decades. A great amount of photometric data is avaliable starting from the first visual and photographic observations collected at the end of the 19th century. The photometric observations prior to 1970 were studied by Haslag (1977) and the main results were summarized by Hall (1976) and Eaton \& Hall (1979). RT Lac, included in the list of close binaries with Ca II $\mathrm{H} \& \mathrm{~K}$ emission by Popper (1942), is a double-lined spectroscopic/eclipsing binary classified as an RS CVn system by Hall (1976). It displays a number of pecularities. In addition to the typical distortion wave its light curve shows substantial variability in shape and in overall brightness both within and outside eclipse. This variability has normally been attributed to the presence of cool, dark starspots on both stars.

Eaton \& Hall (1979) proposed a model in which the moremassive star is so heavily spotted that its average surface flux, despite its higher temperature, is fainter than that of the cooler star, and it is responsible for the intrinsic variability of the

Send offprint requests to: Ö. Çakırlı,

e-mail: cakirli@astronomy.sci.ege.edu.tr system. Moreover, from the long-term broadband photometric monitoring Evren (1989) found that both components are active.

İbanoğlu et al. (2001) presented a detailed mid-term (1993-1999) photometric study of the system deriving the variation of the brightness at mid-eclipses and at maxima and suggested that the variation of the system's brightness mainly arises from the more-massive star. They also suggested that mean brightness of the system shows a cyclic change.

Observations extending over about half a century (19652000, $V$-band) were analysed by Lanza et al. (2002) in the framework of the solar-stellar connection, i.e. by adopting the observed structure of the outer solar atmosphere as a paradigm to interpret stellar observations. According to these authors, the active dark spotted region (with diameter $\sim 40^{\circ}$ ) covers a significant part of the stellar surface occupying low-latitudes (about $0^{\circ}-10^{\circ}$ ) and a possible long-term cycle with a period of approximately $35 \mathrm{yr}$. They found two active longitude zones centered around $0^{\circ}-180^{\circ}$. These resemble the active longitude belts as proposed by Eaton \& Hall (1979) for the RS CVn group in general.

In the present investigation we shall study the long-term $B-$ band photometry of the RT Lac and modelling of the variations of its light curves in the framework of the starspot hypothesis 
based on inverse problem. Our main goal is to study the main characteristics of the photospheric activity such as starspot diameters, locations (e.g. preferred longitudes, latitudes) on each component and short or long-term activity cycles. On the basis of the present results, a possible connection between magnetic activity and orbital period variations is also addressed. The observations are described in Sect. 2. In Sect. 3 we present the procedure of the light-curve analysis. We discuss possible long-term trends in the system brightness and $\mathrm{O}-\mathrm{C}$ residuals in Sect. 4.

\section{Observational data}

The seasonal 23 (1978-2000) photoelectric $B$ and $V$-band light curves of RT Lac were obtained using the $48 \mathrm{~cm}$ Cassegrain telescope at Ege University Observatory, where a monitoring program of RT Lac has been carried out since 1978. All differential magnitudes are relative to the comparison star BD $+43^{\circ}$ 4108 (HD 209219, SAO 51542) adopted by almost all the observers because it is very close to the variable on the celestial sphere and has a similar colour, making negligible the correction for differential atmospheric extinction. BD $+43^{\circ} 4109$ (HD 209220, SAO 51544) was usually adopted as a check star. In Table 1 we present mean time of the observations as year, epochs of primary eclipse, number of observed points $\mathrm{N}$, and references.

Due to the orbital period of the system which is close to an integer number of days, it is diffucult to obtain a good phase coverage of the light curve from a single observing site. Therefore, points which appear to be near in phase could have been obtained after an interval of several weeks or a few months, during which the system might have undergone significant variations. In addition, RT Lac is a very active system and out-of-eclipse light variations up to 0.055 mag have been observed during the course of the same night. Due to the system's intrinsic variability and the unfavourable orbital period some light curves have phase gaps and large intrinsic magnitude scatterings up to $0.06 \mathrm{mag}$. In order to avoid loss of information by averaging such variations, the observations were not averaged into normal points for the analysis. The same weight was given to all points, as adopted by Lanza et al. (2002) in the analysis of $V$-band light curves.

The brightest magnitude from our light-curve sequence turned out to be $m_{B}=8.745 \pm 0.006$ (the magnitudes and colours were transformed to the standard system by using the standard magnitude and colour of the comparison given by Keskin et al. (1994) as $V=7.381, B-V=1.364$ ), at orbital phase 0.25 in the 1985 light curve. This value was assumed to be the magnitude of the system with the spot level at the minimum at first quadrature, thus establishing the magnitude scale calibration for the subsequent light-curve modelling. The overall variation of the system's magnitude versus time was plotted in Fig. 1. In order to give a better display of the long-term variation due to spot activity, only photometric points outside eclipses were plotted (i.e. $0.1 \leq \phi \leq 0.4$ and $0.6 \leq \phi \leq 0.9$ ).

An important issue concerns the specification of the ephemerides adopted to put the observations in phase. The deeper minimum in the light curve was identified by the first
Table 1. B-band light curves of RT Lac.

\begin{tabular}{ccll}
\hline \hline $\begin{array}{c}\text { Light } \\
\text { curve }\end{array}$ & $\begin{array}{c}\text { Epoch of Pri. Min. } \\
\text { HJD }-2400000\end{array}$ & Reference \\
\hline 1978.62 & 43686.05897 & 169 İbanoğlu et al. (1980) \\
1979.62 & 44015.86924 & 192 İbanoğlu et al. (1980) \\
1980.61 & 44431.93418 & 358 Tunca et al. (1983) \\
1981.72 & 44822.62690 & 386 Tunca et al. (1983) \\
1982.76 & 45162.57714 & 280 Evren et al. (1985) \\
1983.70 & 45532.97919 & 202 Evren et al. (1985) \\
1984.57 & 45857.70289 & 357 Evren (1989) \\
1985.62 & 46212.87458 & 508 Evren (1989) \\
1986.56 & 46603.56052 & 179 Evren (1989) \\
1987.75 & 46994.23332 & 191 Evren (1989) \\
1988.65 & 47349.42481 & 377 Evren (1989) \\
1989.59 & 47709.66500 & 434 İbanoğlu et al. (1998a) \\
1990.67 & 48039.47029 & 698 İbanoğlu et al. (1998a) \\
1991.59 & 48435.23327 & 863 İbanoğlu et al. (1998a) \\
1992.57 & 48759.95877 & 511 İbanoğlu et al. (1998a) \\
1993.49 & 49125.28378 & 721 İbanoğlu et al. (2001) \\
1994.65 & 49546.40710 & 136 İbanoğlu et al. (2001) \\
1995.64 & 49921.88943 & 226 İbanoğlu et al. (2001) \\
1996.57 & 50526.76893 & 380 İbanoğlu et al. (2001) \\
1997.64 & 50302.43430 & 394 İbanoğlu et al. (2001) \\
1998.61 & 50982.35541 & 608 İbanoğlu et al. (2001) \\
1999.65 & 51362.89201 & 847 İbanoğlu et al. (2001) \\
2000.66 & 51738.35220 & 423 present paper \\
\hline & &
\end{tabular}

observers as the primary minimum, according to the usual definition adopted for eclipsing binaries, and its orbital phase was assumed to be 0.0 (Haslag 1977). However, such an assumption turned out to be unsatisfactory for RT Lac, because the magnitude at so-defined mid-primary minimum showed large variations with time. The depth of the primary eclipse is sometimes shallower than the secondary minimum, as shown by İbanoğlu et al. (2001) in their Fig. 2. The recent studies by Popper (1991) and İbanoğlu et al. (2001) clarified the cause of this unusual behaviour and they pointed out that the choice of the primary minimum was not appropriate. They found that RT Lac is so heavily spotted, that in most of the light curves the deeper minimum turned out to be the one with the more massive hotter primary in front, as shown by Frasca et al. (2002) also, despite its earlier spectral type. As a consequence of the spot activity, the magnitude at the mid-primary minimum changes remarkably in time because of the large variation of the spotted area on the primary star. Conversely, the magnitude at the center of the secondary minimum appears to be more stable (İbanoğlu et al. 2001). In view of such considerations, it seemed more appropriate to us to define the case when the more massive, hotter component is eclipsed by the less massive, cooler component as the primary minimum, according to the conventional assumption made for eclipsing binaries as well as for the RS CVn systems. An advantage of this choice is that the color index of the system at the deeper primary minimum is redder than at the secondary one, as in other binaries. 


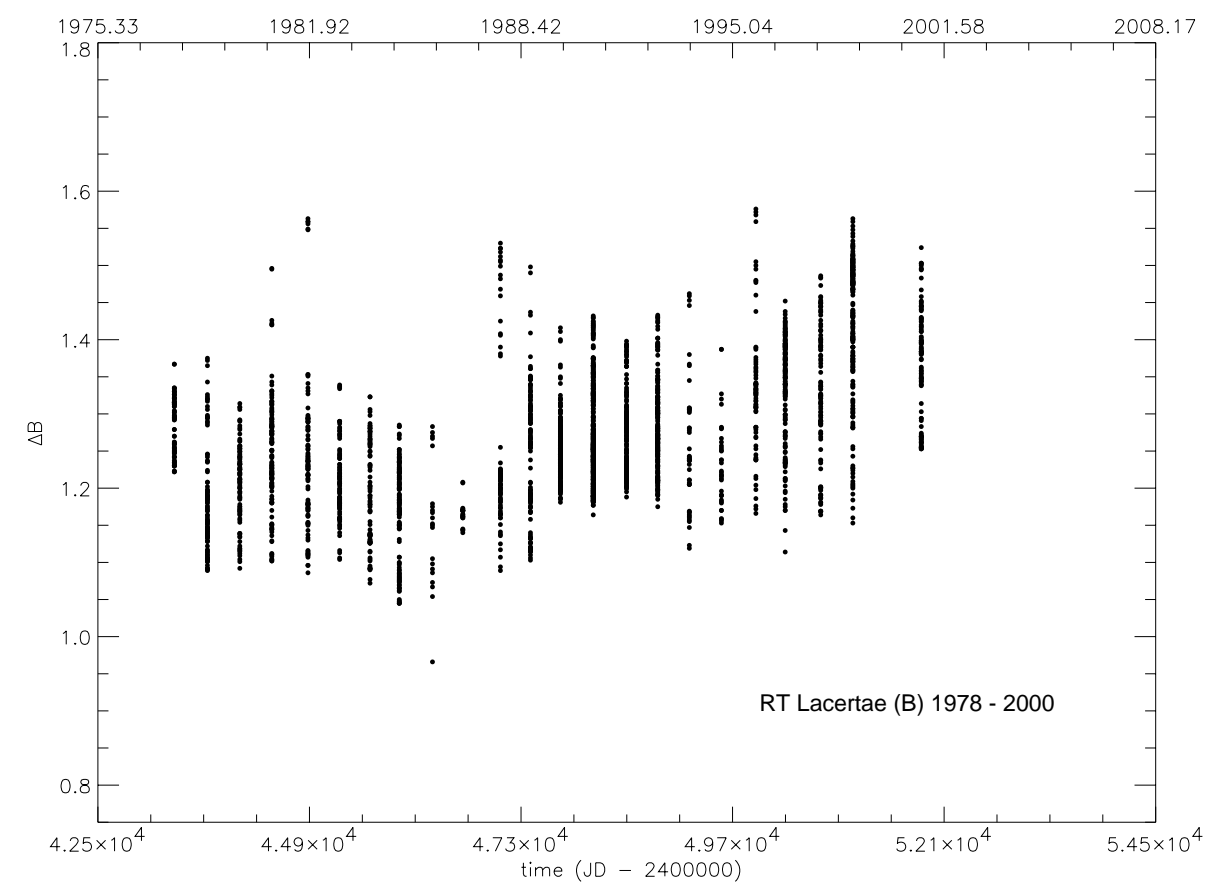

Fig. 1. The magnitude difference $\Delta B$ between the RT Lac and comparison HD 209219 versus time. Only the photometric points outside eclipses are plotted, i.e., with orbital phase $\phi$ in the intervals: $0.1 \leq \phi \leq 0.4$ and $0.6 \leq \phi \leq 0.9$.

\section{Procedure of the light-curve analysis; spot model and the parameters}

To analyse the $B$-light curves of RT Lac, distorted by the presence of spots or spot groups on the components, we used Djurašević's (1992a) pogramme, which is based on the Roche model and the principles arising from the paper by Wilson \& Devinney (1971). The Roche model geometry was implemented according to Kopal (1959) with the Roche potential $\Omega$ at the surface of each star being computed from the mass ratio and star's fractional radius in the direction joining the centers of the two components. Properties of the orbit and the absolute parameters of the components are listed in Table 2. The light-curve analysis was made by applying the inverse-problem method (Djurašević 1992b) based on Marquardt's (1963) algorithm.

As to this method, stellar size in the model is described by the filling factors for the critical Roche lobes $F_{1,2}$ of the primary and secondary component, respectively, which tell us to what degree the stars in the system fill their critical lobes. For synchronous rotation of the components these factors are expressed as the ratio of the stellar polar radii, $R_{1,2}$, and the corresponding polar radii of the critical Roche lobes, i.e., $F_{1,2}=$ $R_{1,2} / R_{\text {Roche }_{1,2}}$. To achieve more reliable estimates of the model parameters in the light-curve analysis programme, we applied a quite dense coordinate grid, having $72 \times 144=10368$ individual elementary cells per star. The intensity and distribution of the radiation of individual cells are determined by the stellar effective temperature, limb-darkening, gravity-darkening and by the reflection effect.

The presence of spotted areas (dark or bright) enable one to explain the asymmetry and the depressions on the light curves of magnetically active RS CVns in connection with RT Lac.
Table 2. Geometrical and physical parameters adopted in modelling the RT Lac light curves.

\begin{tabular}{ll}
\multicolumn{2}{l}{ Orbital elements of the RT Lac } \\
\hline \hline Element \\
\hline$P$ [days] & $\sim 5.074$ \\
Min I [JD Hel] & 2440382.8330 \\
Inclination $i$ [deg] & 89.0 \\
Semi - major axis $a\left[R_{\odot}\right]$ & 15.842 \\
Eccentricity $e$ & 0.0 \\
\hline
\end{tabular}

Stellar and model parameters

\begin{tabular}{lll}
\hline \hline Parameter & Hotter & Cooler \\
\hline$m\left[M_{\odot}\right]$ & 1.582 & 0.624 \\
$R\left[R_{\odot}\right]$ & 4.25 & 4.80 \\
Roche potential & 4.159 & 2.680 \\
$\log g$ & 3.38 & 2.87 \\
Spectrum & $G 5 I V$ & $G 9 I V$ \\
$T_{\text {eff }}[K]$ & 5100 & 4960 \\
$M_{\text {bol }}[\mathrm{mag}]$ & 2.18 & 2.04 \\
\hline
\end{tabular}

In the code the spotted patterns are approximated by circular spots characterized by the temperature contrast of the spot with respect to the surrounding photosphere $\left(A_{\mathrm{s}}=T_{\mathrm{s}} / T_{*}\right)$, by the angular dimension (radius) of the spot $\left(\theta_{\mathrm{s}}\right)$ and by the longitude $\left(\lambda_{\mathrm{s}}\right)$ and latitude $\left(\varphi_{\mathrm{s}}\right)$ of the spot center. The longitude $\lambda_{\mathrm{s}}$ 

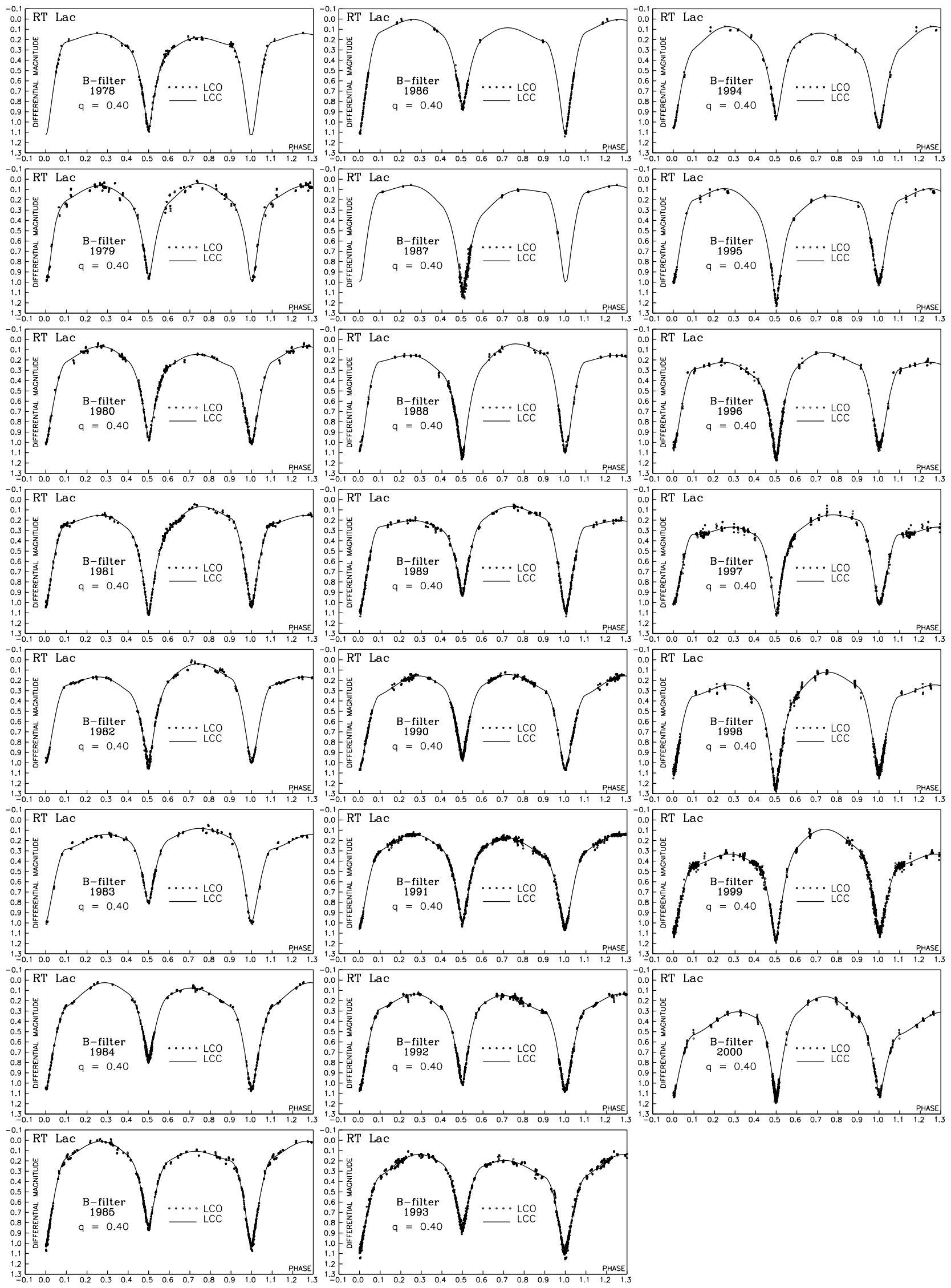

Fig. 2. Observed (LCO) and final synthetic (LCC) light curves of RT Lac obtained by solving inverse problem within the framework of the Roche model with two cool areas on the primary star and one on secondary component. 
is measured clockwise (as viewed from the direction of the $+Z$-axis) from the $+X$-axis (line connecting the stars' centers) in the range $0^{\circ}-360^{\circ}$. The latitude $\varphi_{\mathrm{s}}$ is measured from $0^{\circ}$ at the stellar equator (orbital plane) to $+90^{\circ}$ towards the north $(+Z)$ and $-90^{\circ}$ towards the south $(-Z)$ pole.

For a successful application of this model in the analysis of the sequence of light curves, the method developed by Djurašević (1992b) was used. Optimum model parameters are obtained through the minimization of $S=\Sigma(\mathrm{O}-\mathrm{C})^{2}$, where $\mathrm{O}-\mathrm{C}$ is the residual between the observed and synthetic light curves for a given orbital phase. The minimization of $\mathrm{S}$ is performed in an iterative cycle of corrections of the spot parameters. In this way the inverse-problem method gives us the estimates of spot parameters and their standard errors.

The effective temperature of the unspotted photosphere of the hotter primary was estimated by Popper (1991) to be $\sim 5100 \mathrm{~K}$, using the colour difference between the two components because the spectrum of the more-massive primary is not so well exposed on his plates to allow a determination of its effective temperature. Eaton \& Hall (1979) estimated a difference of $\sim 400 \mathrm{~K}$ between the effective temperatures of two stars, hence we assume an initial value of the effective temperature of $4700 \mathrm{~K}$ for the cooler star. The spot effective temperature was assumed to be about $1000 \mathrm{~K}$ lower than the unspotted photosphere. This spot temperature is an average value for the spots on RS CVn magnetically active components, that were estimated from modelling their infrared light curves. Unfortunately, infrared photometry of RT Lac is quite sparse and has never been analysed to derive the spot temperatures (Milone 1976). In the analysis, the inclination of the orbit was estimated to be $i=89^{\circ} .0$. The present light-curve analysis shows that during the primary minimum the cooler (lessmassive with low-filled Roche lobe) component eclipses the hotter (more-massive) one.

The mass-ratio of the components was fixed to a revised value of $q=m_{\mathrm{c}} / m_{\mathrm{h}}=0.401$ estimated by Frasca et al. (2002) from the REOSC echelle spectrograph in Catania Astrophysical Observatory. From their radial velocity analysis they found the mass of the primary and secondary components as $m_{\mathrm{h}} / M_{\odot}=1.582$ and $m_{\mathrm{c}} / M_{\odot}=0.624$, respectively.

The values of the limb-darkening coefficients were derived from the stellar effective temperature and surface gravity, according to the given spectral type, by using the polynomial proposed by Díaz-Cordovés et al. (1995). During the process of optimisation, with the temperature changes, we have an automatic recomputation of the limb-darkening. Following Lucy (1967), Rucinski (1969) and Rafert \& Twigg (1980), the gravity-darkening coefficients of the stars, $\beta_{\mathrm{h}, \mathrm{c}}$, and their albe$\operatorname{dos}, A_{\mathrm{h}, \mathrm{c}}$, were set at the values of 0.08 and 0.5 , respectively, appropriate for stars with convective envelopes.

Since the stars in the system have convective envelopes, which can exhibit magnetic activity, we started the spotted solution by assuming that the components of RT Lac have cool spots, of the same nature as solar magnetic spots.

Concerning the method it can be said that spot modelling on the components of a close binary system in the frame of the parametric approach has its limitations caused above all by the number of spots, and by their shape and location. By assuming a circular shape of the spot we are able to describe the spot with the minimum number of parameters. The capability of this approach to reliably estimate the locations of the spots on the surface of the component was examined in a number of numerical tests where we were using artificial, model light curves obtained with a priori known parameters of the system and of the spots. In the case of two spots on one of the components these tests proved that the locations of spots can be relatively accurately reconstructed by our method regardless what the initial approximation was. The spot longitude can be quite reliably reconstructed. Since the changes of the spot radius and latitude have a similar impact on the shape of the light curve, their reconstruction is less reliable but still satisfying. If we have more than three spots on the component the reconstruction of their locations is substantially more difficult and less accurate. This is the consequence of the large number of free parameters in the inverse problem. In this way the numerical tests with artificial light curves have shown that the ability of the parameter model is limited by the number of spots on the components above all.

We have performed also some numerical testing with artificial light curves obtained for larger number of small spots randomly distributed over the surface of the components of the system. Such synthetic light curves, normalised to the unspotted light level, were then analysed in the frame of the parameter model with the limited number of spots - active regions (two or three). In solving the inverse problem these synthetic light curves are treated as those observed ones. These numerical experiments show that the inverse problem with the limited, a priori given number of spots (2-3) provides a good fit of such light curve in general. It is clear that the optimum fit cannot describe smaller details on the light curve produced by individual small spots, since now the limited number of larger active regions in some way represent the several smaller spots. These active regions are situated on the positions that correspond approximately to the photocenters of groups of smaller spots. So, the model with the limited number of spots cannot describe the real spot distribution in all the details, but in a rough way it shows the dominant locations and size of active regions whose parameters are determined by the shape of the analysed light curves.

Hence, the results obtained in the frame of the parameter model depend on the chosen working hypothesis about the number of spots on the component, and they reflect also the limitations connected to the idealisation of the parameter approach. The selection and choice of the proper working hypothesis deserves the special attention, and it often forces us to test several options. We have to emphasize that the analysis of real light curves have a number of problems that do not exist in numerical testing with artificial light curves. As the first, there is the problem of the light curve definition, i.e., how well the observations cover all orbital phases, and as the second, there is the problem of signal to noise ratio. These are the problems we have with some of our light curves analysed in this paper. The results obtained in the analysis of the light curves with poor phase sampling should be taken with caution. Even when the model light curve provides a good fit of the observations 
because we do not know the behaviour of the light curve in the phase interval without data.

However, the similar limitations are also present in Doppler imaging method. The Roche model with the limited number of spots (described parametricaly) on the components provides us with the estimates of the basic system parameters and of the locations for the dominant active regions. Doppler imaging technique gives the mapping of the surface of the components. Here we do not have the limitations with the shape and number of active regions, and the resolution and the details of the mapping are dictated by the observational data. Both approaches have their advantages and flaws, and the results depend greatly on the selection of the proper referent light level for the unspotted case.

The preliminary analysis of RT Lac light curves in the period 1978-2000 showed that each light curve in the set is more or less deformed by the presence of spots on the components. The 1979 light curve appears relatively symmetric but the system reached its maximum brightness in 1985 (the orbital phase 0.25 - the maximum after a primary minimum). Although the 1985 light curve exhibits a significant asymmetry, this value of the brightest magnitude was assumed to be the magnitude of the minimum spotted activity of the system at first quadrature, thus establishing the magnitude scale calibration for the subsequent light curve modelling.

The complexity of the task forced us to perform the lightcurve analysis in several steps. First, all light curves were normalized to the reference maximum light level of the 1985 light curve at the orbital phase 0.25 . After that we made the lightcurve analysis with optimisation in the system and spot parameters. Moreover, since the results depend on the choice of the adopted working hypothesis, the present analysis was carried out within the framework of several hypotheses with spotted areas on the components. In the preliminary analysis we considered several different configurations of spots on the components. A satisfactory fit of the observations was obtained with the hypothesis of two active spotted regions on the more massive component and one spotted region on the less massive secondary one. Thus, this was the working hypothesis we used for optimization in all free parameters of the inverse problem (system and spot parameters) for well defined light curves. The results showed that the basic parameters of the system (filling factors, inclination of the orbit and temperature of the secondary component) are different from one light curve to another, which was expected. Namely, all light curves are deformed by the presence of spots which makes the estimation of the basic parameters of the system difficult. A simultaneous determination of these parameters together with the parameters of spots can lead to errors in both sets of parameters. So, we adopted average values of basic parameters of the system obtained in the simultaneous analysis of all light curves. We do not see any physical reason for the change of the basic system parameters in the relatively short time interval (1978-2000) covered by the observations. Thus, we fixed them (see Table 2) and reanalysed the light curves this time for the spot parameters. In this case we obtained a very good fit of the observations for the whole set of the analysed light curves. The obtained results (see Table 3) indicate that the complex nature of the light-curve variations during the examined period could be almost entirely explained by the changes in the parameters of the spotted areas.

\section{Results}

\subsection{Light curve fitting and spot parameters}

The spot parameters (angular radius, $\theta^{\mathrm{s}}$ longitude, $\lambda^{\mathrm{s}}$ and latitude, $\varphi^{\mathrm{S}}$ ) derived from this light curve analysis are listed in Table 3. The determination of these parameters is based on a simultaneous fitting of the available light curves in the $B$ band for different epochs of observations with the same set of basic system parameters. The first four columns of the Table 3 present the year, the number of observations $n$, the final sum of squares of residuals between observed and synthetic light curves $\sum_{i=1}^{n}\left(\mathrm{O}_{i}-\mathrm{C}_{i}\right)^{2}$, and the standard deviation of the residuals $\sigma=\sqrt{\frac{\sum_{i=1}^{n}\left(\mathrm{O}_{i}-\mathrm{C}_{i}\right)^{2}}{(n-1)}}$. The errors of the parameters' estimates arise from the nonlinear least-squares method, on which the inverse-problem method is based.

Using the inverse-problem solutions for each seasonal light curves, Fig. 2 presents the optimum fit of the observed light curves (LCO) by the synthetic ones (LCC). The final O-C residuals between the observed (LCO) and optimum synthetic (LCC) light curves are given in the Fig. 3.

\subsection{General evolution of the spot features}

A view of the Roche model of RT Lac obtained with the parameters estimated by analysing the seasonal light curves is provided in Fig. 4. The view of the system is shown for orbital phases 0.25 and 0.75 for each seasonal light curve, so one can follow how the spots are evolving over time. By this continuous evolution of the spot distribution over the surface of RT Lac we can describe the seasonal changing shape of the light curves.

The total area covered by spots was calculated by $S_{\text {spot-area }}=\left[\frac{S_{11}+S_{12}+S_{2} * k^{2}}{1+k^{2}}\right]$, where $S_{11}$ refers to the first spot on the more-massive primary, $S_{12}$ refers to the second spot on the same star, $S_{2}$ refers to the spot on the secondary star and k is the ratio of the fractional radii of the stars, $k=r_{\text {cool }} / r_{\text {hot }}$. The spot areas on the more massive primary and less massive secondary are plotted in Fig. 5a, and the total spotted area is shown in Fig. 5b. Due to the smaller signal to noise ratio in the 1979 observations the observed magnitudes show large scattering. On the other hand, the 1987 light curve has large gaps. Therefore, the total spotted areas we derived are less reliable for those light curves, as it will be expected from the high sigma, given in Table 3. The areas covered by $S_{11}$ and $S_{12}$ plotted against the time in Fig. 6a. Figure $6 \mathrm{~b}$ display the percentage of the spotted area of the cooler component. Two cool spots were also shown on the surface of the hotter, more massive primary component. The spots derived for the 1986 and 1987 light curves on the uneclipsed region of the primary are smeared in latitude as the photometric information alone is insufficient to defining their exact locations. Also in some cases the phase coverage of the light curve is not complete, (cf. 1995) making any spots that appear in the missing regions less reliable.

The total spotted area as a part of the stellar surface disk on the more massive primary varies from $18 \%$ to $47 \%$ and for the secondary $9 \%$ to $21 \%$ in accordance with our model. 
Table 3. Results of the analysis of the RT Lac (1978-2000) B-filter photometrical observations obtained by solving the light curve inverse problem for the Roche model with spotted areas on the components.

\begin{tabular}{|c|c|c|c|c|c|c|c|c|c|c|c|c|c|}
\hline Year & $n$ & $\Sigma(\mathrm{O}-\mathrm{C})^{2}$ & $\sigma$ & $\theta_{\mathrm{h}}^{S_{1}}$ & $\lambda_{\mathrm{h}}^{S_{1}}$ & $\varphi_{\mathrm{h}}^{S_{1}}$ & $\theta_{\mathrm{h}}^{S_{2}}$ & $\lambda_{\mathrm{h}}^{S_{2}}$ & $\varphi_{\mathrm{h}}^{S_{2}}$ & $\theta_{\mathrm{c}}^{S_{3}}$ & $\lambda_{\mathrm{c}}^{S_{3}}$ & $\varphi_{\mathrm{c}}^{S_{3}}$ & $L_{\mathrm{h}} /\left(L_{\mathrm{h}}+L_{\mathrm{c}}\right)$ \\
\hline \multirow[t]{2}{*}{1978} & 169 & 0.0451 & 0.0163 & 57.0 & 95.5 & 67.5 & 40.2 & 227.8 & -48.7 & 38.1 & 313.2 & 49.3 & 0.428 \\
\hline & & & & \pm 0.3 & \pm 1.1 & \pm 1.3 & \pm 0.3 & \pm 0.9 & \pm 0.7 & \pm 0.7 & \pm 2.4 & \pm 1.3 & - \\
\hline \multirow[t]{2}{*}{1979} & 192 & 0.2030 & 0.0325 & 27.7 & 351.1 & 2.2 & 37.7 & 195.4 & -51.5 & 18.0 & 80.7 & 3.7 & 0.455 \\
\hline & & & & \pm 1.3 & \pm 3.2 & \pm 10.9 & \pm 0.8 & \pm 3.6 & \pm 1.3 & \pm 1.2 & \pm 6.7 & \pm 22.2 & - \\
\hline \multirow[t]{2}{*}{1980} & 358 & 0.1076 & 0.0173 & 37.9 & 355.6 & 53.2 & 57.3 & 184.9 & -81.6 & 32.7 & 261.5 & 56.6 & 0.424 \\
\hline & & & & \pm 0.9 & \pm 2.0 & \pm 3.0 & \pm 0.3 & \pm 6.1 & \pm 0.4 & \pm 0.7 & \pm 2.1 & \pm 1.6 & - \\
\hline \multirow[t]{2}{*}{1981} & 386 & 0.1056 & 0.0165 & 33.6 & 348.5 & 49.8 & 46.8 & 194.3 & -47.6 & 32.1 & 79.7 & 28.9 & 0.448 \\
\hline & & & & \pm 0.7 & \pm 1.7 & \pm 1.6 & \pm 0.2 & \pm 0.9 & \pm 0.3 & \pm 0.4 & \pm 0.9 & \pm 1.8 & - \\
\hline \multirow[t]{2}{*}{1982} & 280 & 0.1253 & 0.0212 & 57.2 & 38.4 & 72.2 & 35.9 & 163.5 & -49.6 & 21.0 & 97.8 & 46.7 & 0.418 \\
\hline & & & & \pm 0.4 & \pm 2.7 & \pm 0.7 & \pm 0.3 & \pm 1.9 & \pm 0.5 & \pm 1.2 & \pm 4.4 & \pm 4.2 & - \\
\hline \multirow[t]{2}{*}{1983} & 202 & 0.0600 & 0.0172 & 50.5 & 42.3 & 53.6 & 16.8 & 112.4 & -4.2 & 22.0 & 249.0 & -10.4 & 0.448 \\
\hline & & & & \pm 0.6 & \pm 1.8 & \pm 1.1 & \pm 0.7 & \pm 2.9 & \pm 13.1 & \pm 0.6 & \pm 2.2 & \pm 12.1 & - \\
\hline \multirow[t]{2}{*}{1984} & 357 & 0.1068 & 0.0173 & 38.7 & 12.6 & 43.6 & 23.8 & 284.7 & -12.4 & 17.7 & 17.4 & 38.9 & 0.457 \\
\hline & & & & \pm 0.6 & \pm 1.5 & \pm 1.3 & \pm 0.6 & \pm 2.3 & \pm 5.3 & \pm 0.4 & \pm 3.7 & \pm 1.4 & - \\
\hline \multirow[t]{2}{*}{1985} & 508 & 0.1714 & 0.0184 & 31.6 & 16.4 & 45.0 & 20.2 & 170.8 & -43.7 & 38.5 & 274.0 & 48.5 & 0.491 \\
\hline & & & & \pm 0.6 & \pm 1.6 & \pm 1.6 & \pm 0.3 & \pm 4.5 & \pm 1.1 & \pm 0.4 & \pm 1.1 & \pm 1.3 & - \\
\hline \multirow[t]{2}{*}{1986} & 179 & 0.0355 & 0.0141 & 16.8 & 13.8 & 45.4 & 31.4 & 207.5 & -57.3 & 24.9 & 311.8 & 7.1 & 0.474 \\
\hline & & & & \pm 1.5 & \pm 4.0 & \pm 8.5 & \pm 0.4 & \pm 2.2 & \pm 0.7 & \pm 0.4 & \pm 0.8 & \pm 7.2 & - \\
\hline \multirow[t]{2}{*}{1987} & 191 & 0.3040 & 0.0399 & 16.6 & 77.5 & 6.4 & 39.4 & 178.9 & -40.8 & 38.8 & 247.9 & 54.2 & 0.480 \\
\hline & & & & \pm 3.8 & \pm 27.5 & \pm 38.4 & \pm 0.4 & \pm 4.4 & \pm 0.7 & \pm 1.3 & \pm 3.8 & \pm 2.7 & - \\
\hline \multirow[t]{2}{*}{1988} & 377 & 0.1183 & 0.0177 & 52.5 & 98.0 & 63.4 & 43.3 & 190.2 & -55.2 & 25.1 & 358.4 & 55.3 & 0.420 \\
\hline & & & & \pm 0.2 & \pm 0.7 & \pm 0.8 & \pm 0.2 & \pm 1.2 & \pm 0.3 & \pm 0.5 & \pm 7.0 & \pm 1.0 & - \\
\hline \multirow[t]{2}{*}{1989} & 434 & 0.1223 & 0.0168 & 54.9 & 74.0 & 66.4 & 16.5 & 123.7 & -4.9 & 50.7 & 282.5 & 85.2 & 0.473 \\
\hline & & & & \pm 0.3 & \pm 1.1 & \pm 0.6 & \pm 0.3 & \pm 1.5 & \pm 21.9 & \pm 0.3 & \pm 5.8 & \pm 0.8 & - \\
\hline \multirow[t]{2}{*}{1990} & 698 & 0.1265 & 0.0135 & 57.2 & 31.2 & 57.2 & 47.2 & 156.6 & -72.6 & 45.9 & 278.8 & 64.6 & 0.425 \\
\hline & & & & \pm 0.2 & \pm 0.7 & \pm 0.4 & \pm 0.1 & \pm 1.3 & \pm 0.2 & \pm 0.2 & \pm 1.0 & \pm 0.5 & - \\
\hline \multirow[t]{2}{*}{1991} & 863 & 0.1662 & 0.0139 & 51.4 & 353.9 & 49.8 & 53.2 & 132.5 & -64.2 & 47.1 & 263.7 & 64.1 & 0.427 \\
\hline & & & & \pm 0.2 & \pm 0.5 & \pm 0.5 & \pm 0.2 & \pm 0.6 & \pm 0.3 & \pm 0.2 & \pm 0.8 & \pm 0.3 & - \\
\hline \multirow[t]{2}{*}{1992} & 511 & 0.1288 & 0.0159 & 57.3 & 38.1 & 62.6 & 29.8 & 164.3 & -33.9 & 36.5 & 290.0 & 34.4 & 0.439 \\
\hline & & & & \pm 0.3 & \pm 1.2 & \pm 0.5 & \pm 0.2 & \pm 1.2 & \pm 0.6 & \pm 0.2 & \pm 0.5 & \pm 0.7 & - \\
\hline \multirow[t]{2}{*}{1993} & 721 & 0.3611 & 0.0224 & 56.8 & 24.2 & 49.9 & 18.2 & 130.6 & -13.8 & 53.5 & 267.8 & 57.7 & 0.473 \\
\hline & & & & \pm 0.4 & \pm 0.9 & \pm 0.7 & \pm 0.4 & \pm 1.4 & \pm 5.2 & \pm 0.3 & \pm 0.7 & \pm 0.8 & - \\
\hline \multirow[t]{2}{*}{1994} & 136 & 0.0418 & 0.0175 & 48.5 & 7.3 & 44.0 & 23.9 & 154.8 & -9.8 & 47.5 & 270.9 & 62.3 & 0.473 \\
\hline & & & & \pm 1.0 & \pm 1.9 & \pm 3.0 & \pm 0.3 & \pm 2.0 & \pm 4.1 & \pm 0.6 & \pm 2.0 & \pm 1.4 & - \\
\hline \multirow[t]{2}{*}{1995} & 226 & 0.0813 & 0.0190 & 36.1 & 177.6 & -35.6 & 57.3 & 25.4 & 79.4 & 34.0 & 261.7 & 46.8 & 0.429 \\
\hline & & & & \pm 0.2 & \pm 2.6 & \pm 0.6 & \pm 0.5 & \pm 5.7 & \pm 0.7 & \pm 0.8 & \pm 2.0 & \pm 2.4 & - \\
\hline \multirow[t]{2}{*}{1996} & 380 & 0.1817 & 0.0219 & 56.3 & 36.9 & 69.2 & 57.3 & 144.3 & -58.9 & 36.1 & 288.8 & 57.3 & 0.396 \\
\hline & & & & \pm 0.4 & \pm 2.2 & \pm 0.7 & \pm 0.2 & \pm 0.8 & \pm 0.3 & \pm 0.6 & \pm 1.7 & \pm 2.0 & - \\
\hline 1997 & 394 & 0.2637 & 0.0259 & 57.3 & 72.2 & 47.9 & 57.2 & 199.0 & -73.7 & 34.2 & 254.9 & 55.0 & 0.392 \\
\hline & & & & \pm 0.4 & \pm 1.1 & \pm 0.8 & \pm 0.4 & \pm 3.6 & \pm 0.5 & \pm 0.8 & \pm 2.5 & \pm 1.7 & - \\
\hline 1998 & 608 & 0.4442 & 0.0270 & 57.1 & 347.8 & 65.4 & 42.2 & 191.9 & -32.7 & 50.9 & 85.4 & 53.4 & 0.442 \\
\hline & & & & \pm 0.6 & \pm 2.6 & \pm 0.9 & \pm 0.2 & \pm 1.1 & \pm 0.4 & \pm 0.3 & \pm 0.7 & \pm 1.3 & - \\
\hline 1999 & 847 & 0.5484 & 0.0254 & 56.9 & 6.6 & 53.6 & 56.5 & 166.0 & -59.9 & 50.7 & 86.4 & 57.3 & 0.415 \\
\hline & & & & \pm 0.3 & \pm 0.9 & \pm 0.6 & \pm 0.2 & \pm 1.4 & \pm 0.3 & \pm 0.2 & \pm 0.7 & \pm 0.7 & - \\
\hline 2000 & 423 & 0.2616 & 0.0249 & 55.2 & 358.1 & 34.8 & 57.3 & 208.7 & -56.8 & 50.5 & 82.6 & 36.5 & 0.422 \\
\hline & & & & \pm 0.5 & \pm 0.9 & \pm 1.5 & \pm 0.2 & \pm 0.7 & \pm 0.3 & \pm 0.4 & \pm 0.8 & \pm 1.2 & - \\
\hline
\end{tabular}

Note: $n$ - number of observations, $\Sigma(\mathrm{O}-\mathrm{C})^{2}-$ final sum of squares of residuals between observed (LCO) and synthetic (LCC) light curves, $\sigma$ - standard deviation of the observation, $\theta^{\mathrm{S}_{1,2,3}}, \lambda^{\mathrm{S}_{1,2,3}}$ and $\varphi^{\mathrm{S}_{1,2,3}}-$ spots' angular dimensions, longitudes and latitudes (in arc degrees) and $L_{\mathrm{h}} /\left(L_{\mathrm{h}}+L_{\mathrm{c}}\right)$ - luminosity of the hotter star (including spots on the components).

The relative uncertainties of the total spotted area $S_{\text {spot-area }}$ varies from $\sim 8 \%$ to $35 \%$ for the whole system.
The overall spotted areas behaviour on both components indicates a long-term change, suggesting a possible long-term 


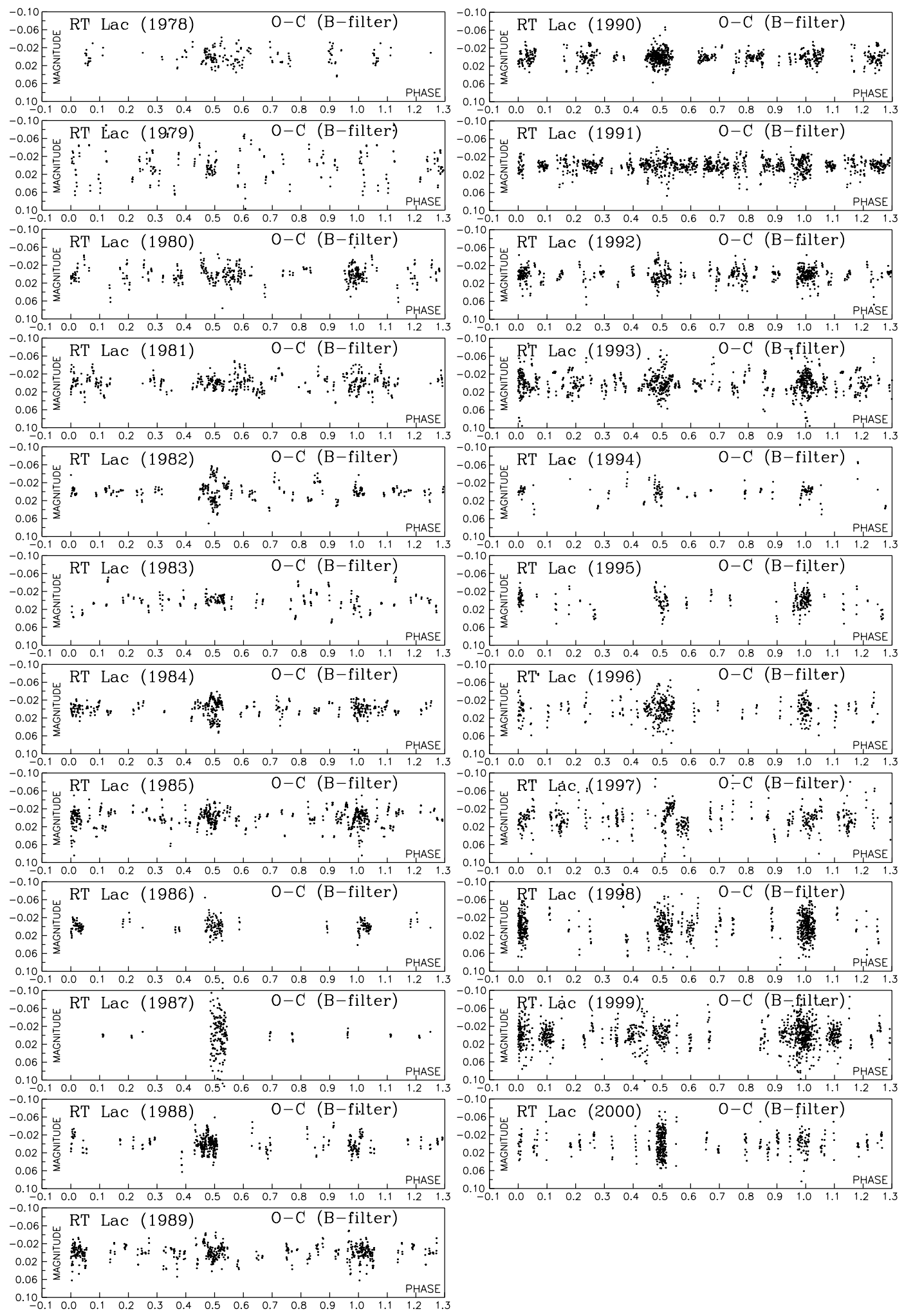

Fig. 3. Final O-C residuals obtained by solving the light curves using the inverse problem within the framework of the Roche model with spotted areas on both stars.

cycle with a period of approximately 33 yr. Maximum was observed around 2000, while a minimum was reached around 1985 which we used as a reference light level for normalizing the light curves during the analysis of sequence of light curves. A Fourier analysis based on the Scargle periodogram
(Scargle 1982) was applied in the search for shorter term periodicity. We found a period of $8.4 \mathrm{yr}$ in the variation of spotted area vs. time (see Fig. 5). On the other hand, the periodogram analysis did not show any significant periodicity in the spot area size for the G5IV and G9IV star separately. We do not expect 

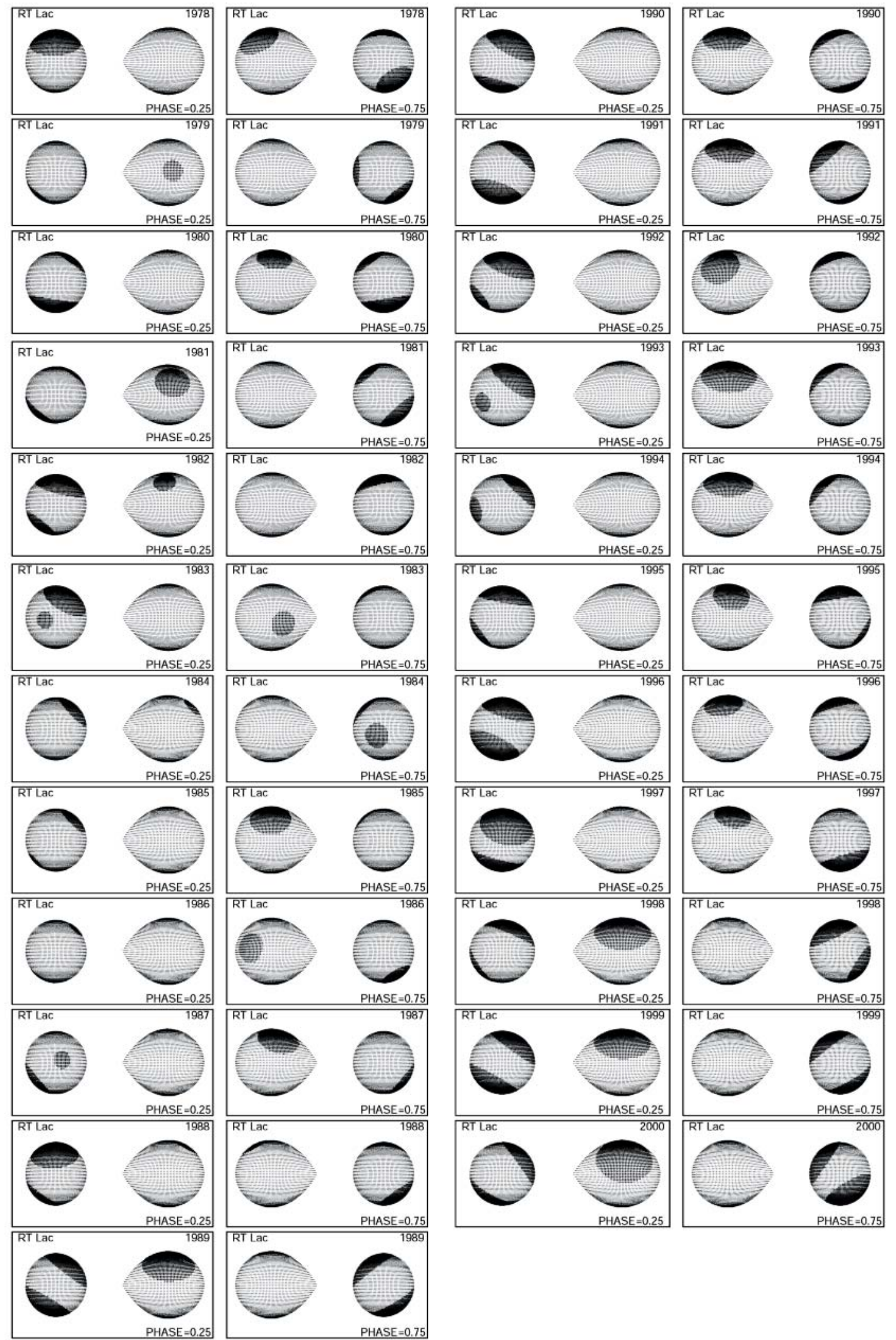

Fig. 4. The view of the Roche model for RT Lac at orbital phases 0.25 and 0.75 obtained with parameters estimated by the light-curve analysis.

any significant periodicity in G9IV star according to İbanoğlu et al. (2001), because when the cool component faces the observer the light variability is at the lowest level and shows no periodicity. However, the total spotted area on the secondary is smaller in the 1978-1985 period, and larger between 19852000. The total spotted area on the more massive primary (see 
Fig. 5a, squared symbols) indicates the existence of an activity cycle of about $9 \mathrm{yr}$.

Our long-term sequence of data allows us to investigate the change of the longitudinal distribution of the spotted area on each component of RT Lac. Figure 6a, obtained from the analysis of the light curves, shows spots located generally on two active longitude zones at $\sim 45^{\circ}$ and $\sim 170^{\circ}$ on the primary star, as marked with dotted line. We find evidence for a sudden longitudinal change for the spot located at $\sim 45^{\circ}$ near 1979,1991 and 2000. Moreover, the active longitude region at $\sim 170^{\circ}$ seems to shift towards the smaller longitudes as time elapsed. We have no evidence for the possible existence of a flip-flop mechanism of active longitudes, as seen in some RS CVn stars (cf. Jetsu et al. 1991; Berdyugina \& Tuominen 1998; Taş et al. 1999; Henry et al. 1995) such as EI Eri, II Peg and MM Her.

\subsection{Orbital period variation and long-term trends}

RT Lac has been observed by many investigators since its discovery in 1908 by M. L. Ceraski. Studies of O-C diagrams have been performed by several authors including Hall (1991), Applegate (1992), Keskin et al. (1994) and recently İbanoğlu et al. (2001). The latter presented also the latest O-C diagram of the system (cf. their Fig. 6). It shows a long-term modulation with a period of approximately one century upon which a shorter term modulation is superimposed. The timings for minimum light obtained photolectrically were derived by İbanoğlu et al. (2001) with a standard deviation of $\sim 0.0016 \mathrm{~d}$. The timedependent behaviour of the $\mathrm{O}-\mathrm{C}$ change was attributed to the light-time effect and magnetic activity (İbanoğlu et al. 2001). They derived the third-body orbit and subtracting the lighttime effect from all O-Cs they obtained an approximately sinusoidal modulation with an amplitude of $0.028 \mathrm{~d}$ and a period of $17.8 \mathrm{yr}$. It is interesting to study the relationship between such a short-time modulation with the change of spotted area.

The link between cyclic magnetic activity and orbital period change was proposed by Applegate (1992) with the later revision and refinements by Lanza et al. (1998b) and Lanza \& Rodonó (1999). These authors propose that the orbital period modulation can be explained as coupling of the binary orbit to changes in the gravitational quadrupole moment of the magnetically active star in the system as it goes through an activity cycle. One of the testable predictions of the Applegate's model is that the extrema of the long-term light curves and the $\mathrm{O}-\mathrm{C}$ curve should coincide (see e.g. Hall 1991).

In order to search for any relationship between the change in the brightness of the system that could be caused by the effects of spots, we compare the $\mathrm{O}-\mathrm{C}$ diagram with the total spotted area. The $\mathrm{O}-\mathrm{C}$ diagram includes times of primary and secondary eclipses in $B, V$ and $R$ bands, constructed according to the revised ephemerides by İbanoğlu et al. (2001). Only the timings obtained photoelectrically between 1978 and 2000 were used, visual and photographic timings were not taken into account.

In Fig. 7a we have shown all the O-Cs for mid-eclipses obtained up to 2001 and their representation with the light-time effect. This effect is subtracted from photoelectrically obtained
Table 4. Orbital period variation of RT Lac.

\begin{tabular}{cccc}
\hline \hline Sector & $\begin{array}{c}\text { Time Interval } \\
\text { (HJD - 2 400 000) }\end{array}$ & $\begin{array}{c}P_{\text {ref }} \\
\text { 5.073982 }\end{array}$ & $\Delta P$ \\
\hline I & $43782.00-46286.00$ & 5.073972 & -0.86 \\
II & $46286.00-48171.00$ & 5.074002 & $+1^{8} .73$ \\
III & $48171.00-49612.00$ & 5.073942 & -3.46 \\
IV & $49612.00-51091.00$ & 5.074042 & +5.18 \\
V & $51091.00-51870.00$ & 5.073982 & -8.64 \\
\hline
\end{tabular}

O-Cs and the residuals are plotted in Fig. 7c for the time interval 1978-2000. In Fig. 7b we plot the total spotted area in order to see the existence of any correlation between orbital period change and magnetic activity. As the total spotted area is decreasing the $\mathrm{O}-\mathrm{Cs}$ are also decreasing and vice versa. As seen from Fig. $7 b$ the total spotted area shows at least two decrements and two increments. Similar behaviour is also seen for the orbital change. When the spotted area is decreasing the orbital period of the system is shortening. The orbital periods have been calculated for five time intervals and are given in Table 4. Both time intervals, in which orbital period has been changed, are different and the amount of the period change is also different from one time interval to another. On the contrary, the last two light curves show that the areas covered by spots are maximum, while the orbital period of the system seems to be at its shortest. The cyclical change in both Figs. 7b and 7c seems closely connected each other. While the mean cycle obtained from the total spotted area is about $8.7 \mathrm{yr}$, the $\mathrm{O}-\mathrm{Cs}$ change gives a mean cycle of $9.7 \mathrm{yr}$.

\section{Discussion and conclusions}

We have used an inverse-problem technique based on Marquardt's algorithm for the analysis of sequence of light curves of the RS CVn-type binary RT Lac. Our analysis of an extended sequence of light curves of RT Lac has led to several interesting results.

The results obtained in this study (Table 3 and corresponding figures) indicate that the synthetic light curves obtained by solving the inverse problem in the case of the Roche model with two dark spotted regions on the primary and one on the secondary fits the observations very well (almost within the measurement accuracy). The light curve variations may be produced mainly due to change of the position and size of the spotted areas on the primary. The spot activity on the more massive primary is localized in two main active regions around longitudes $\sim 45^{\circ}$ and $\sim 170^{\circ}$. There may be some tendency for activity to switch alternatively from one active longitude to the other from time to time, but the phenomenon is not clearly apparent as in, e.g., II Peg or other RS CVn binaries (Berdyugina \& Tuominen 1998).

The light curves obtained in 1980, 1990 and 2000 show a greater spot coverage on the primary. Moreover, it seems that maximum spotted area lasts about 3-4 yr. In 1985 the magnetic activity of the primary seems to be at a minimum. In contrast, the 1999 and 2000 light curves show that the spotted area on 


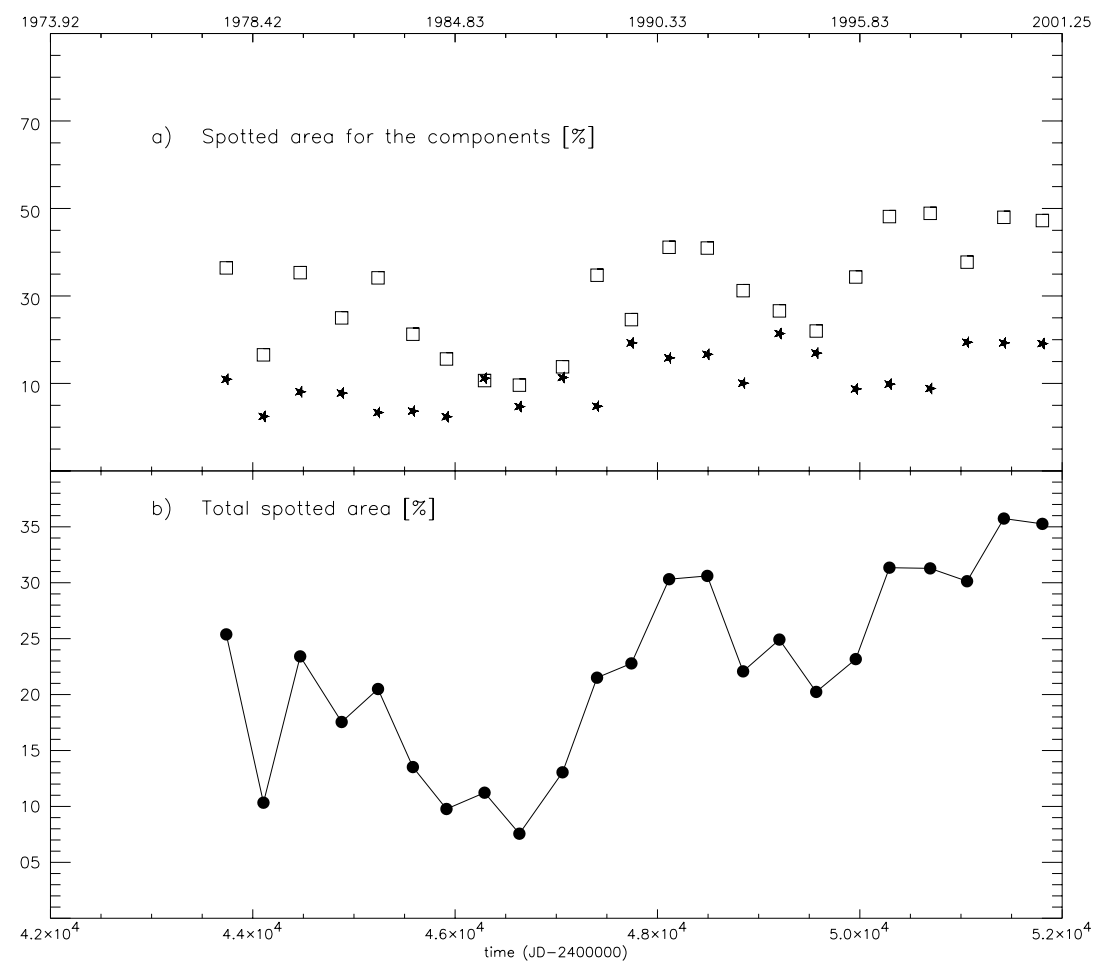

Fig. 5. a) The evolution of the spotted area vs. time on the more massive primary and the less massive secondary. Squares: spotted area of the more massive component. Stars: spotted area of the secondary component and b) The total spotted area of the whole system.

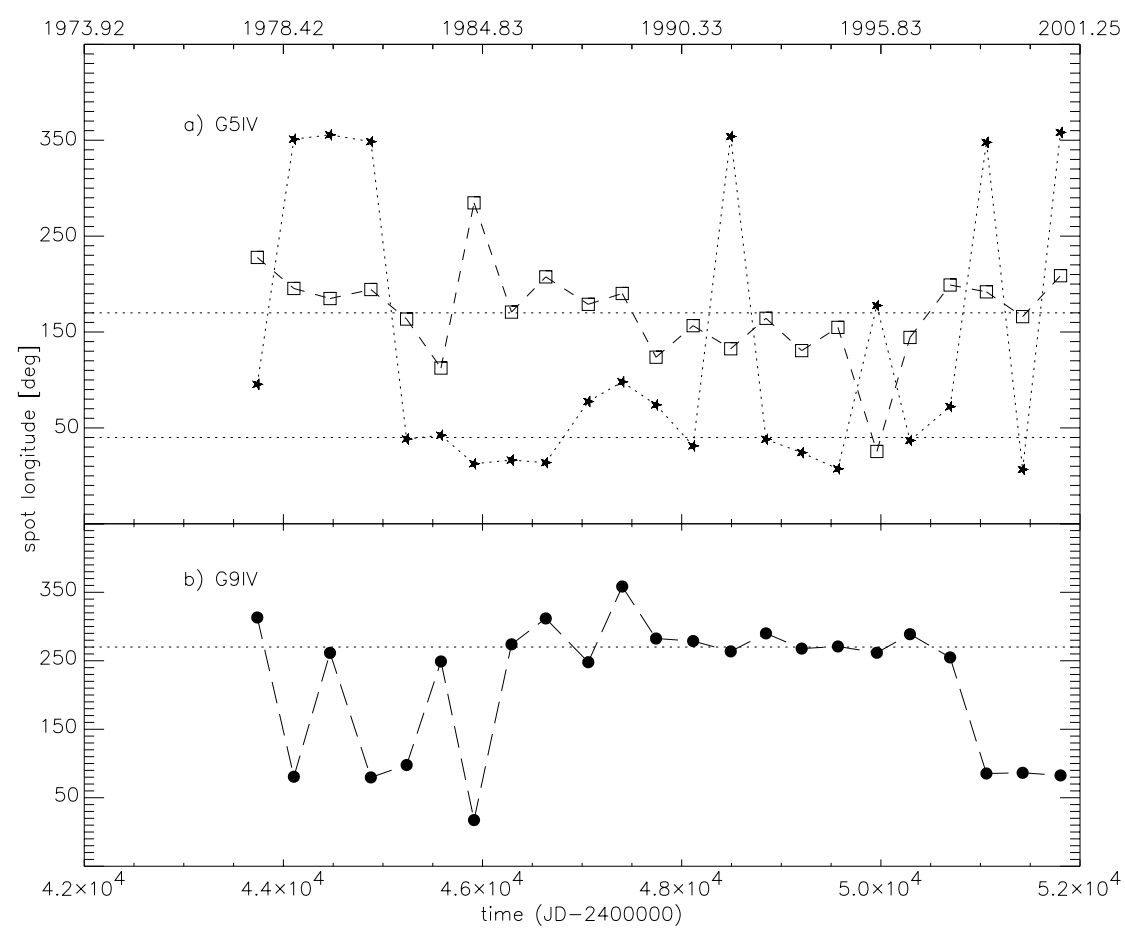

Fig. 6. Longitude vs. time (JD) for spots on a) (G5IV) primary and b) for secondary (G9IV). Star like symbols refer to the longitudinal change of the first spot with time on the primary. Squared symbols refer to the longitudinal change of the second spot on the primary denoted in the text as $S_{12}$. Filled circles refer to longitudinal change of the spot against the time on the secondary, denoted as $S_{2}$ in text.

the primary star was increased and reached at least two times compared to that of secondary. These results are in agreement with those obtained spectroscopically by Frasca et al. (2002), who conclude that the primary component is at least ten times more active than that of secondary in 2000. 


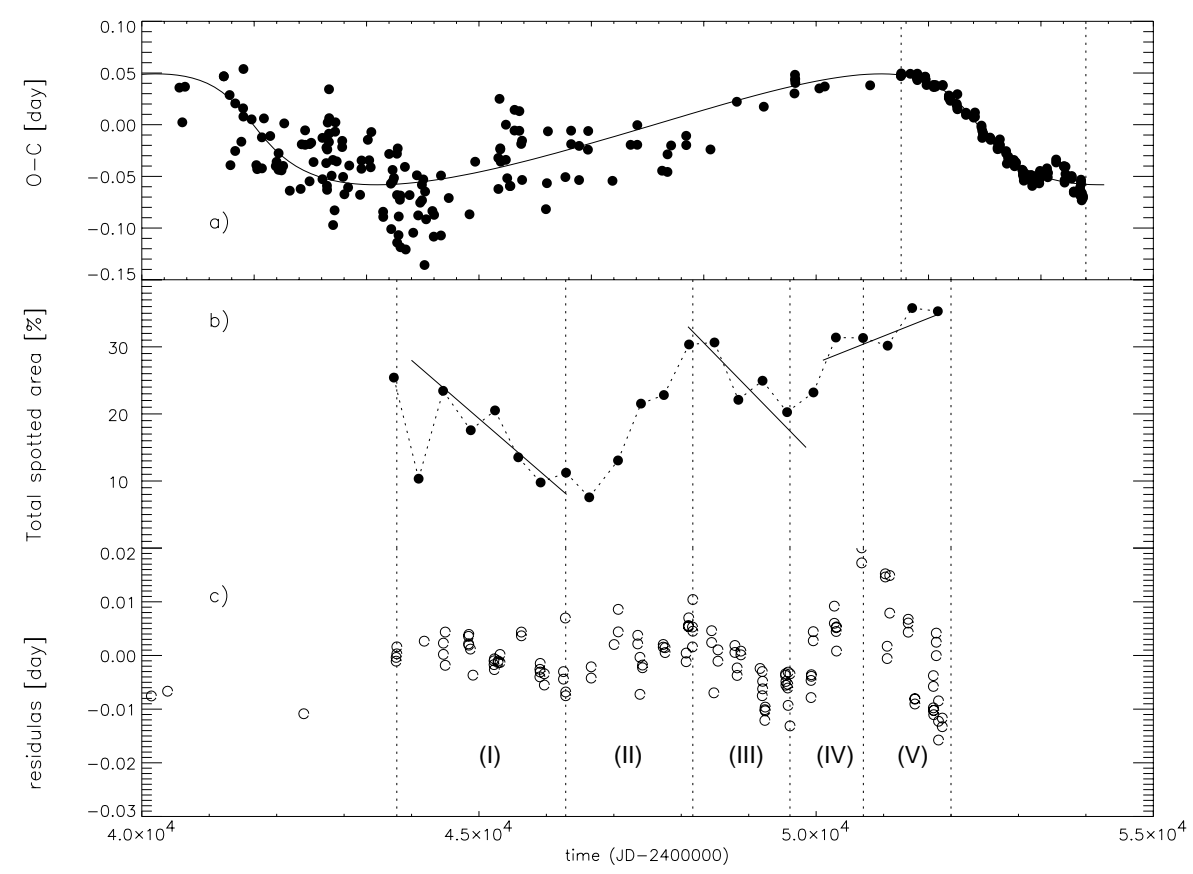

Fig. 7. a) Residuals for all the times of minimum light of RT Lac with respect to the linear light elements. The continuous curve represents the light-time effect. b) The total spotted area, as in Fig. 5b, for the whole system. c) Residuals, between the O-Cs and the light-time effect. The O-Cs between two dashed-lines as obtained from 1978-2001 (top panel) has been divided five intervals at the bottom panel. The mean orbital period was calculated for each segment.

Extensive photometric $V$-data of the RT Lac have been analysed for spot modelling by Lanza et al. (2002). They studied the light curves under the conditional technique of Maximum Entropy (Gull \& Skilling 1984; Vogt et al. 1987) and Tikhonov regularizations (Piskunov et al. 1990) by using the $\chi^{2}$ minimization. They proposed two active regions on the primary; the first is at $0^{\circ}$ and the second at $180^{\circ}$.

In this paper, in the analysis of the light curves we used a parametric approach to determine the distribution and size of spots in the Roche model. Parametric models have certain limitations connected to the idealization in the spot definition and characteristics. Increased number of free parameters make the inverse problem difficult to solve and can lead to nonunique solutions. This imposes certain restrictions on the number of spots on the components. The tests performed with different initial spot distribution on the components of the system proved that the solutions obtained in this paper are relatively stable, i.e., the iterative procedure converges to given solutions regardlessly of initial value and location of spots. Of course, this does not imply that the real distribution of active regions on the component stars is in complete conformity with the obtained solutions, but we expect the dominant spots to be on the estimated locations. The estimate of the basic system parameters presents a real problem also because in the analysed set of the RT Lac light curves there is none that is not deformed by the presence of spots. The way we have chosen to solve this problem (average values of basic system parameters) seems logical and we give the basic parameters obtained in this way a larger weight compared to solutions obtained for individual light curves.

Lanza et al. (2002) used a different approach to map the stellar surface which is based on the Maximum Enthropy method and Tikhonov regularizing criteria. In essence their results are in good agreement with ours. The differences are caused by the approximations used and by the procedure of light-curve normalization also. Namely, their reference light curve is relatively symmetric 1979 light curve, while we normalize the light curves to the maximum brightness level of the system in 1985.

The orbital period change of the RT Lac system seems to be closely connected to the magnetic activity of the components. As the spot coverage is increased the orbital period of the system gets longer. This relationship has been revealed at least four time intervals. However, we should note that the large amount of period decrease in the time interval 1997-2000 seems to be in the opposite direction.

Acknowledgements. Ö. Çakırlı thanks to Koç VAKFI for his educational-background supporting. We would like to thank the refree for useful suggestions and comments. Financial support of the Ege University Science Fund under the project $98 /$ Fen/056 is gratefully acknowledged. This research has made use of the Simbad database, operated at the CDS, Strasbourg, France, and supported by the Ministry for Sciences and Technology of Serbia through the project 1191 "Stellar physics".

\section{References}

Applegate, J. H. 1992, ApJ, 385, 621

Berdyugina, S. V., \& Tuominen, I. 1998, A\&A, 336, L25

Diaz-Cordovès, J., Claret, A., \& Gimènez, A. 1995, A\&AS, 110, 329

Djurašević's, G. 1992a, Ap\&SS, 196, 241

Djurašević's, G. 1992b, Ap\&SS, 197, 17

Eaton, J. A., \& Hall, D. S. 1979, ApJ, 227, 907

Evren, S. 1989, Ap\&SS, 161, 303 
Evren, S., Tunca, Z., İbanoğlu, C., \& Tümer, O. 1985, Ap\&SS, 108, 383

Frasca, A., Çakırlı, Ö., Catalano, C., et al. 2002, A\&A, 388, 298

Hall, D. S. 1976, in IAU Colloq. 29, Multiple Periodic Variable Stars, Part I, ed. W. S. Fitch (Dordrecht: Reidel), 287

Hall, D. S. 1991, in The Sun and Cool Stars: Activity, Magnetism, Dynamos, ed., I. Tuominen, D. Moss, \& G. Rüdiger (Berlin: Springer-Verlag), 353

Haslag, K. P. 1977, Master Thesis, Vanderbilt University, Nashville, Tennessee

Henry, G. W., Eaton, J. A., Hammer, J., \& Hall, D. S. 1995, Ap\&SS, 229,185

Gull, S. F., \& Skilling, J. 1984, in Indirect Imaging, ed. J. A. Roberts (Cambridge: Cambridge Univ. Press), 267

İbanoğlu, C., Evren, S., Taş, G., Devlen, A., \& Çakırlı, Ö. 2001, A\&A, 371,626

İbanoğlu, C., Kurutaç, M., Tümer, O., et al. 1980, Ap\&SS, 72, 61

İbanoğlu, C., Pekünlü, E. R., Keskin, V., Tunca, Z., \& Akan, M. C. 1998, Ap\&SS, 257, 11

Jetsu, L. P., Tuominen, I., \& Nations, H. 1991, in The Sun and Cool Stars: Activity, Magnetisim, Dynamos, ed. I. Tuominen, D. Moss, \& G. Rüdiger (Berlin: Springer-Verlag), 381
Keskin, V., İbanoğlu, C., Akan, M. C., Evren, S., \& Tunca, Z. 1994, A\&A, 287, 817

Kopal, Z. 1959, Close Binary Systems (London: Chapman, \& Hall) Lanza, A. F., Rodonò, M., \& Rosner, R. 1998b, MNRAS, 296, 893

Lanza, A. F., \& Rodonò, M. 1999, A\&A, 349, 887

Lanza, A. F., Catalano, S., Rodonò, M., et al. 2002, A\&A, 386, 583

Lucy, L. B. 1967, Zs. f. Ap., 89

Marquardt, D. W. 1963, J. Soc. Ind. Appl. Math., 11, No. 2, 431

Milone, E. F. 1976, ApJS, 31, 93

Piskunov, N. E., Tuominen, I., \& Vilhu, O. 1990, A\&A, 230, 363

Popper, D. M. 1942, PASP, 10, 292

Popper, D. M. 1991, AJ, 101, 220

Rafert, J. B., \& Twigg, L. W. 1980, MNRAS, 139, 78

Rucinski, S. M. 1969, Acta Astr., 19, 245

Scargle, J. D. 1982, ApJ, 263, 835

Taş, G., Evren, S., \& İbanoğlu, C. 1999, A\&A, 349, 546

Tunca, Z., İbanoğlu, C., Tümer, O., Ertan, A. Y., \& Evren, S. 1983, Ap\&SS, 93, 431

Vogt, S. S., Penrod, G. D., \& Hatzes, A. P. 1987, ApJ, 321, 496

Wilson, R. E., \& Devinney, E. J. 1971, ApJ, 166, 605 